\title{
Adipocytes play an etiological role in the podocytopathy of high-fat diet-fed rats
}

\author{
Jinn-Yang Chen 1,2, Deng-Yuan Jian3,4, Chih-Chan Lien³, Yu-Ting Lin3, \\ Ching-Heng Ting ${ }^{3}$, Luen-Kui Chen ${ }^{3}$, Ting-Chia Hsu³, Hsuan-Min Huang ${ }^{3}$, Yu-Ting Wu ${ }^{3}$, \\ Tse-Ting Kuan3, Yu-Wen Chao5,6, Liang-Yi Wu7, Seng-Wong Huang ${ }^{2}$ and \\ Chi-Chang Juan $3,5,8$
}

1Division of Nephrology, Department of Medicine, Taipei Veterans General Hospital, Taipei, Taiwan 2Faculty of Medicine, National Yang-Ming University, Taipei, Taiwan 3Institute of Physiology, National Yang-Ming University, Taipei, Taiwan

${ }^{4}$ Division of Nephrology, Wen-Lin Hemodialysis Unit, Taipei, Taiwan

${ }^{5}$ Department of Medical Research and Education, Taipei Veterans General Hospital, Taipei, Taiwan

${ }_{6}^{6}$ Section of Nephrology, Department of Internal Medicine, Heping Branch, Taipei City Hospital, Taipei, Taiwan 7Department of Bioscience Technology, College of Science, Chung Yuan Christian University, Chung Li District, Taoyuan City, Taiwan

${ }^{8}$ Department of Education and Research, Taipei City Hospital, Taipei, Taiwan

\author{
Correspondence \\ should be addressed \\ to C-C Juan \\ Email \\ ccjuan@ym.edu.tw
}

\begin{abstract}
Obesity is a risk factor that promotes progressive kidney disease. Studies have shown that an adipocytokine imbalance contributes to impaired renal function in humans and animals, but the underlying interplay between adipocytokines and renal injury remains to be elucidated. We aimed to investigate the mechanisms linking obesity to chronic kidney disease. We assessed renal function in high-fat (HF) diet-fed and normal diet-fed rats, and the effects of preadipocyte- and adipocyte-conditioned medium on cultured podocytes. HF diet-fed and normal diet-fed Sprague Dawley rats were used to analyze the changes in plasma BUN, creatinine, urine protein and renal histology. Additionally, podocytes were incubated with preadipocyte- or adipocyte-conditioned medium to investigate the effects on podocyte morphology and protein expression. In the HF diet group, $24 \mathrm{~h}$ urinary protein excretion $(357.5 \pm 64.2 \mathrm{mg} /$ day vs $115.9 \pm 12.4 \mathrm{mg} / \mathrm{day}$, $P<0.05)$ and the urine protein/creatinine ratio were significantly higher $(1.76 \pm 0.22$ vs $1.09 \pm 0.15, P<0.05)$, increased kidney weight $(3.54 \pm 0.04 \mathrm{~g}$ vs $3.38 \pm 0.04 \mathrm{~g}, P<0.05)$ and the glomerular volume and podocyte effacement increased by electron microscopy. Increased renal expression of desmin and decreased renal expression of CD2AP and nephrin were also seen in the HF diet group $(P<0.05)$. Furthermore, we found that adipocyte-conditioned medium-treated podocytes showed increased desmin expression and decreased CD2AP and nephrin expression compared with that in preadipocyte-conditioned medium-treated controls $(P<0.05)$. These findings show that adipocyte-derived factor(s) can modulate renal function. Adipocyte-derived factors play an important role in obesity-related podocytopathy.
\end{abstract}

\section{Key Words}

- adipocytes

- adipocytokine

- high-fat diet

- podocytopathy

- visceral fat
Journal of Endocrinology (2016) 231, 109-120
๑) 2016 Society for Endocrinology Printed in Great Britain
Published by Bioscientifica Ltd. 


\section{Introduction}

Overweight and obesity now constitute a global public health crisis. They associate with metabolic syndrome, abdominal obesity, glucose intolerance, insulin resistance, lipid profile abnormalities and elevated blood pressure (Cerezo et al. 2013). The dominant risk factors for metabolic syndrome are abdominal obesity and insulin resistance. Epidemiological studies have revealed a significant association between insulin resistance and obesity, pathological hyperplasia and/or hypertrophy of adipose tissue (Galic et al. 2010). Adipose tissue is an important source of metabolically active secretory products, including free fatty acids, leptin, tumor necrosis factor- $\alpha$ (TNF- $\alpha$ ), interleukin-6 (IL-6), plasminogen activator inhibitor-1, resistin and adiponectin, which can lead to the development of cardiovascular diseases (Galic et al. 2010, Van de Voorde et al. 2013).

An imbalance of adipokines in patients with chronic kidney disease (CKD) is a consequence of impaired renal function, abnormal hormone plasma levels and altered regulatory mechanisms caused by the uremic milieu (Mills et al. 2013). Leptin, a small peptide $(16 \mathrm{kDa})$ belonging to the IL-6 family of cytokines, acts as a coactivator with transforming growth factor (TGF)- $\beta$ to promote renal tubulointerstitial fibrosis (Kümpers et al. 2007). Adiponectin, another small peptide $(30 \mathrm{kDa})$ secreted almost entirely by adipocytes, is the strongest predictor of stages 3-5 CKD according to the K/DOQI guidelines, showing a significant nonlinear inverse association (Nanayakkara et al. 2009). Resistin is an independent factor for CKD in nondiabetic obese patients (Stępień et al. 2013). Recent studies have established that visceral fat associates with cardiometabolic risk caused by dysregulation of adipokine secretion and the associated inflammatory state (Després et al. 2008, Phillips \& Prins 2008).

The strong clinical association between metabolic syndrome and the risk of renal impairment is well recognized (Locatelli et al. 2006, Ninomiya \& Kiyohara 2007, Heo et al. 2010). Several studies have shown that obesity carries an increased risk of CKD and development of end-stage renal disease (ESRD) (Gelber et al. 2005, Obermayr et al. 2008, Satirapoj et al. 2013). CKD is defined as the presence of microalbuminuria (Locatelli et al. 2006) and a decrease in the glomerular filtration rate to $<60 \mathrm{~mL} / \mathrm{min}$ per $1.73 \mathrm{~m}^{2}$, which is calculated using the abbreviated modification of diet in the renal disease equation (Levey et al. 1999).
In addition, there is a high prevalence of insulin resistance in nondiabetic CKD patients, both in children and adults (Pham et al. 2011, Canpolat et al. 2012), and in chronic dialysis patients (Hung \& Ikizler 2011), and its etiology is complex and multifactorial. It is unclear whether insulin resistance is a cause or a consequence of $\mathrm{CKD}$, and more convincing mechanistic evidence is needed to clarify this issue.

Regardless of the human or experimental model of glomerular damage, podocyte loss associates with progressive glomerular diseases, and glomerular volume acts as a key determinant of the total glomerular filtration surface (Fukuda et al. 2012, Weil et al. 2012). Podocytes serve as the final defense against the loss of serum albumin, and podocyte injury is closely related to proteinuria (Mathieson 2011). A reduction in podocyte number and density has been suggested to play an important role in the progression of podocyte dysfunction and the development of proteinuria (Tharaux \& Huber 2012). Desmin is a sensitive marker for acute podocyte damage in the glomerulus (Herrmann et al. 2012) and glomerular podocyte damage during the early stages of diabetic nephropathy (Kakimoto et al. 2014). In kidney podocytes, the slit diaphragm proteins nephrin and CD2-associated protein (CD2AP) contribute to the filtration barrier, which prevents the loss of urinary proteins (Asanuma et al. 2007, Xiao et al. 2013). Knight and coworkers found that the expression of glomerular nephrin expression decreases and the urinary albumin level increases in a high-fat (HF) diet model of obesity. Furthermore, hypertension and renal inflammation, which was detected as an increase in renal macrophage-specific CD68-positive staining, were present in the HF diet-fed animals (Knight et al. 2010). Recently, obesity was shown to lead to decreased adiponectin levels, resulting in podocyte injury (Sharma 2009). However, the relationship between obesity and podocyte function has not been fully evaluated.

Body weight gain has been shown to be an independent risk factor for CKD (Nguyen \& Hsu 2007, Hsu et al. 2009). In addition, studies have shown that the development of obesity-related CKD is mediated by cardiovascular risk factors (Foster et al. 2008, Munkhaugen et al. 2009). Although there have been several large epidemiological studies of obesity-related CKD, the association between obesity and CKD and the underlying interplay between adipocytokines and renal injury remain to be elucidated. The purpose of this study was to clarify the mechanism linking obesity to CKD.

Published by Bioscientifica Ltd 


\section{Materials and methods}

\section{Study design}

Male Sprague Dawley rats, weighing 200-250g, were housed four to a cage in an air-conditioned room $\left(22 \pm 2^{\circ} \mathrm{C}\right)$ on a $12 \mathrm{~h}$ light cycle (06:00-18:00h). Except when scheduled for an oral glucose tolerance test (OGTT) or killing, the animals had free access to food and water. The rats in the HF diet group $(n=8)$ were fed a HF diet (60\% fat), while those in the control $(\mathrm{C} ; n=8)$ were fed standard chow. The experiment lasted for 3 months, and changes in the levels of plasma glucose, insulin, insulin sensitivity, triglycerides, cholesterol, blood urea nitrogen (BUN), creatinine, urine creatinine and albumin, as well as the albumin/creatinine ratio, were measured. Insulin sensitivity was measured using the OGTT. After the 3-month experimental period, the body fat distribution of each rat was evaluated by computed tomography (CT). The animals were then killed, the fat pad weight was measured and adipocytes were isolated for the analysis of adipocyte size and size distribution. We removed both kidneys from each rat and measured their weight. We then removed the kidney cortex for histological analysis and Western blotting. We calculated the mean glomerular volume by the point counting method at a magnification of $400 \times$ on hematoxylin and eosin-stained sections as described previously (Adamczak et al. 2003). In addition, the levels of nephrin, CD2AP and desmin in the kidneys were measured by Western blotting. All procedures were carried out in accordance with the Taiwan Government's Guide for the Care and Use of Laboratory Animals. The research protocol was approved by the Animal Welfare Committee of the National Yang-Ming University.

\section{Adipose tissue and kidney histology}

Epididymal white adipose tissues were removed, fixed in $4 \%$ neutral formaldehyde, dehydrated and embedded in paraffin for serial sectioning of tissue blocks at a thickness of $4 \mu \mathrm{m}$. The sections were stained with hematoxylin (Muto Pure Chemicals Co, Ltd, Bunkyuo-ku, Tokyo, Japan) and eosin (Merck). Three random fields in each section from each rat were examined under a microscope.

The kidney cortex was removed and processed for light microscopy. The specimens were fixed in 10\% formaldehyde and embedded in paraffin for serial sectioning of tissue blocks at a thickness of $4 \mu \mathrm{m}$. The sections were cut perpendicular to the outer border of the kidney (the cortex area) and stained with hematoxylin and eosin, periodic acid-Schiff reagent and an antibody against desmin (Santa Cruz Biotechnology). The stained slides were examined under a microscope, with the reviewers blinded to the treatment group, and the calculated results were normalized to glomerular volume. For histological analysis, we measured eight glomeruli in each field (magnification, 200x). Three random fields in each section were chosen, and five sections from each rat were used for analysis. Representative images were captured using a digital spot camera. The glomerular volume was calculated as the total area of the glomerular tuft $\left(\mathrm{A}_{\mathrm{T}}\right) \times(1.5) \times 1.382 / 1.01 \quad(1.382=$ shape coefficient, $1.01=$ size distribution coefficient) (Adamczak et al. 2003).

\section{Cell cultures}

Conditionally immortalized mouse podocytes, kindly provided by Prof. Jochen Reiser, MD, PhD (Rush University Medical Center, Chicago, IL, USA), were cultured as described previously (Mundel et al. 1997). These immortalized mouse podocytes can be cultured under different conditions to generate two different phenotypes; under permissive conditions $\left(33^{\circ} \mathrm{C}\right)$, they proliferate and maintain an epithelial phenotype with a cobblestonelike morphology (immature podocytes), whereas under nonpermissive conditions $\left(37^{\circ} \mathrm{C}\right)$, they stop proliferation and undergo conversion to arborized cells (differentiated or mature podocytes). This differentiation results in the sprouting of long processes from the cell bodies and in the formation of spindle-like projections from these primary processes.

In this study, differentiated podocytes were cultured at $37^{\circ} \mathrm{C}$ for $8-10$ days without IFN- $\gamma$ in Dulbecco's modified Eagle's (DME) medium containing $5.5 \mathrm{mmol} / \mathrm{L}$ glucose, 100 units $/ \mathrm{mL}$ penicillin, $100 \mu \mathrm{g} / \mathrm{mL}$ streptomycin (all from Gibco BRL) and 10\% fetal bovine serum (Biological Industries, Kibbutz Beit $\mathrm{Ha}^{\prime}$ Emek, Israel) in $5 \% \mathrm{CO}_{2}$ and $95 \%$ humidified air. The podocytes were plated at $0.2 \times 10^{6}$ cells/well on 24-well plates (Corning) and serum-starved overnight. They were incubated for $24 \mathrm{~h}$ with conditioned medium from 3T3-L1 preadipocytes or 3T3-L1 adipocytes, prepared as described below and washed twice with phosphate-buffered saline; RNA was isolated for real-time polymerase chain reaction (RT-PCR) studies.

3T3-L1 preadipocytes (American Type Culture Collection) were seeded on 6-well plates (Falcon, Becton Dickinson, NJ, USA) and grown in complete medium (DME high glucose $(25 \mathrm{mmol} / \mathrm{L})$ medium containing $100 \mathrm{U} / \mathrm{mL}$ penicillin, $100 \mu \mathrm{g} / \mathrm{mL}$ streptomycin, and $10 \%$ fetal bovine serum) in $10 \% \mathrm{CO}_{2}$, with the medium replaced

Published by Bioscientifica Ltd 
every 3 days. After the cells reached confluency by 3 days, they were induced to differentiate for 3 days in complete medium containing $0.5 \mathrm{mM}$ isobutylmethylxanthine, $0.5 \mu \mathrm{M}$ dexamethasone and $1.7 \mu \mathrm{M}$ insulin (all from Sigma). The medium was then replaced with complete medium, which was changed every 3 days until the cells were fully differentiated. By day $10,>95 \%$ of the preadipocytes differentiated into adipocytes as determined by staining for lipid accumulation using Oil Red O. For the collection of conditioned medium, 3T3-L1 preadipocytes or well-differentiated 3T3-L1 adipocytes were incubated for $24 \mathrm{~h}$ in serum-free DME medium containing $5.5 \mathrm{mmol} / \mathrm{L}$ glucose and $1 \%$ bovine serum albumin. Cultured podocytes were incubated with preadipocyte-cultured medium (PCM), adipocytecultured medium (ACM) or adipocyte-cultured medium with an antibody against adiponectin (PA1-054, Thermo Fisher Scientific), leptin (5367-100, BioVision), resistin (sc-22315, Santa Cruz Biotechnology), TNF- $\alpha$ (NB600587, Novus Biologicals, Littleton, CO, USA) or the IgG isotype for $24 \mathrm{~h}$. Podocyte function was then evaluated in vitro. Podocyte injury was evaluated by the expression of nephrin, CD2AP and desmin in the slit diaphragm, which was measured using RT-PCR.

\section{Computer tomography}

For each rat, CT was performed on one slice from each of the three regions, namely, the sacroiliac joint (inguinal level), the upper portion of the iliac crest (L4-L5, upper iliac crest level) and $1.5 \mathrm{~cm}$ above the second slice (L1-L2, kidney level) as described previously with minor modifications (Hillebrand et al. 2010) using the ultra-high resolution setting of a SOMATOM Sensation 16 scanner (Siemens AG). A density range between -150 and -40 Hounsfield units was used to define an area of fat, and a range between -40 and 250 Hounsfield units was used to define an area of muscle (water density $=0$ ). All CT scans were performed in the prone position under anesthesia. The total adipose and muscle tissue in these three regions was calculated using a computer. The intra-abdominal cavity, including the retroperitoneal space, was outlined manually with a cursor and the area of adipose tissue was expressed as a percentage of the total area calculated. Subtraction of the intra-abdominal area from the total abdominal area gave the subcutaneous area. The average visceral fat area/subcutaneous fat area ratio in the slices from the three regions was used for statistics.

\section{Oral glucose tolerance test}

Rats were subjected to an OGTT after overnight fasting. A 0 min blood sample was taken from each rat, and the rats were immediately given a glucose solution (concentration, $0.2 \mathrm{~g} / 0.1 \mathrm{~mL} ; 0.1 \mathrm{~mL} / 100 \mathrm{~g}$ body weight) by gavage, and four additional blood samples were collected at 30, 60, 90 and $120 \mathrm{~min}$. The concentration of plasma insulin was determined using a commercial rat insulin enzyme-linked immunosorbent assay (ELISA) kit (Mercodia AB, Uppsala, Sweden), and the plasma glucose level was measured using a glucose analyzer (Model 23A, Yellow Springs Instrument, Yellow Springs, OH, USA).

\section{Biochemical measurements}

The levels of plasma total cholesterol, high-density lipoprotein cholesterol, low-density lipoprotein cholesterol, triglycerides and free fatty acids were measured using commercial EIA kits from Diagnostic Systems GmbH (Holzheim, Germany). The levels of plasma BUN and creatinine were measured using kits from ARKRAY, Inc. (Minami-ku, Kyoto, Japan). Plasma data of leptin, adiponectin and TNF- $\alpha$ levels were measured using ELISA kits purchased from R\&D Systems.

\section{Enzyme-linked immunosorbent assay}

Samples were collected in $0.1 \mathrm{M} \mathrm{HCl}$ buffer and mixed by pipetting. After centrifugation $(1000 \boldsymbol{g}, 10 \mathrm{~min})$, the supernatant was decanted and collected for measurement of the intracellular cGMP concentration in accordance with the product specification.

\section{Transmission electron microscopy}

A fragment of the kidney cortex was cut into $1 \mathrm{~mm}^{3}$ pieces and fixed in $2.5 \%$ glutaraldehyde in Millonig solution, and then embedded in PolyBed 812 (Polysciences, Inc, Warrington, PA, USA) for transmission electron microscopy (TEM) analysis (Sharma et al. 2008).

\section{Western blotting}

Whole cell lysates were prepared by sonication in ice-cold lysis buffer (1\% Triton X-100, $50 \mathrm{mM} \mathrm{KCl,}$ $25 \mathrm{mM}$ HEPES, pH 7.8, supplemented with $10 \mu \mathrm{g} / \mathrm{mL}$ leupeptin, $20 \mu \mathrm{g} / \mathrm{mL}$ aprotinin, $125 \mu \mathrm{M}$ dithiothreitol, $1 \mathrm{mM}$ phenylmethylsulfonyl fluoride and $1 \mathrm{mM}$ sodium

Published by Bioscientifica Ltd 
orthovanadate), and the protein concentration of the lysates was determined using a Bio-Rad protein assay (BioRad Laboratories). Samples containing $30 \mu \mathrm{g}$ of protein were boiled for $5 \mathrm{~min}$ in $50 \mu \mathrm{L}$ of reducing sample buffer, and the proteins were resolved by $12.5 \%$ SDS-PAGE for $90 \mathrm{~min}$ at 160 volts. The proteins were then transferred to a polyvinylidene difluoride membrane at $60 \mathrm{~V}$ for $120 \mathrm{~min}$. The membrane was blocked for $1 \mathrm{~h}$ at room temperature in blocking buffer ( $5 \%$ skimmed milk in $10 \mathrm{mM}$ Tris, $100 \mathrm{mM}$ $\mathrm{NaCl}, 0.1 \%$ Tween 20, pH 7.5) and then immunoblotted for $24 \mathrm{~h}$ at $4^{\circ} \mathrm{C}$ with a primary antibody in blocking buffer, followed by $1 \mathrm{~h}$ at room temperature with horseradish peroxidase-conjugated secondary antibodies in blocking buffer. The bound antibodies were revealed using chemiluminescent reagent (Amersham Biosciences). To detect multiple signals from a single membrane, the membrane was treated with stripping buffer $(59 \mathrm{mM}$ Tris-HCl, 2\% SDS, 0.75\% 2-mercaptoethanol, $\mathrm{pH}$ 6.8) for $50 \mathrm{~min}$ at $50^{\circ} \mathrm{C}$ before reblotting with a different primary antibody. The signals were normalized to those for $\beta$-actin. Goat polyclonal antibodies against nephrin or desmin, a mouse monoclonal antibody against avian $\beta$-actin (all from Santa Cruz Biotechnology) and a rabbit polyclonal antibody against CD2AP (Novus Biologicals, Littleton, CO, USA) were used. Horseradish peroxidase-conjugated goat anti-rabbit IgG, goat anti-mouse IgG and donkey anti-goat IgG secondary antibodies were purchased from Santa Cruz Biotechnology.

Table 1 Body weight, fat pad weight, kidney weight, metabolism-related biochemical parameters and lipid profiles in controls (C) and high-fat diet-fed rats (HF) $(n=8)$.

\begin{tabular}{|c|c|c|c|}
\hline Parameters & C & HF & $P$-value \\
\hline Body weight (g) & $459 \pm 9$ & $532 \pm 22$ & $<0.05$ \\
\hline \multicolumn{4}{|l|}{ Fat pad weight (g) } \\
\hline Epididymal & $8.5 \pm 0.9$ & $22.2 \pm 2.5$ & $<0.05$ \\
\hline eritoneal & $10.7=$ & $31.5 \pm$ & $<0.05$ \\
\hline Ingu & 12.2 & 23. & $<0$ \\
\hline eight (g) & $3.38 \pm$ & $3.54 \pm$ & $<0.05$ \\
\hline & $115 \pm$ & $138.8 \pm$ & $<0.05$ \\
\hline$)$ & 24. & $45.5 \pm$ & $<0.05$ \\
\hline Tric & $0 . \varepsilon$ & 0.1 & $<0.05$ \\
\hline ol (mg/dL) & $145.8 \pm 8.9$ & $244.7 \pm 9.1$ & $<0.05$ \\
\hline BUN (mg/dL) & $12.4 \pm 0.5$ & $12.3 \pm 0.8$ & NS \\
\hline & $0.23 \pm 0.02$ & $0.32 \pm 0.01$ & $<0.05$ \\
\hline tein (mg/day) & $115.9 \pm 12.4$ & $357.5 \pm 64.2$ & $<0.05$ \\
\hline Urine creatinine (mg/day) & $120.4 \pm 19.8$ & $202.2 \pm 18.9$ & $<0.05$ \\
\hline Urine protein:creatinine & $1.09 \pm 0.15$ & $1.76 \pm 0.22$ & $<0.05$ \\
\hline
\end{tabular}

Values are expressed as mean \pm s.D. Statistical analysis was performed using Mann-Whitney $U$ test with repeated measurements. A value of $P<0.05$ was considered significant.

BUN, blood urea nitrogen; NS, not significant.

\section{Fluorescent microscopy}

To investigate the adipokine effects of PCM or ACM, differentiated podocytes cultured on $12 \mathrm{~mm}$ cover slips in a 24-well plate were treated with PCM or ACM with the IgG isotype as the control or with an antibody against adiponectin, leptin, resistin or TNF- $\alpha$. The immunofluorescent staining of desmin (green), nephrin (green), CD2AP (green), F-actin (red) and DAPI (blue) was detected using a Leica DM6000B fluorescent microscope (Leica Microsystems). Each slide was evaluated in eight randomly selected fields from four slides per sample (magnification, 100x) by two different investigators who were blinded to the sample identities.

\section{Quantitative analysis of desmin, nephrin and CD2AP transcripts by RT-PCR}

Total RNA was extracted from cultured mouse podocytes using TRI Reagent (Sigma-Aldrich). The extraction was carried out as described previously (Seow et al. 2011). TaqMan Gene Expression Assays (FAM dye-labeled MGB probe; Applied Biosystems) containing specific primers (assay ID for desmin: Mm00802455_m1; nephrin: Mm00497828_m1; CD2AP: Mm01277622_g1; GAPDH: Mm99999915_g1), the TaqMan MGB probe (FAM dyelabeled), TaqMan Fast Universal PCR Master Mix and $100 \mathrm{ng}$ of cDNA were used to detect and quantify the mRNA levels of desmin, nephrin and CD2AP in cultured mouse podocytes. GAPDH served as the internal control. The Ct value of GAPDH was subtracted from that of desmin, nephrin and CD2AP $(\Delta \mathrm{Ct})$. The $\Delta \mathrm{Ct}$ value of the ACM group was compared with that of the PCM group.
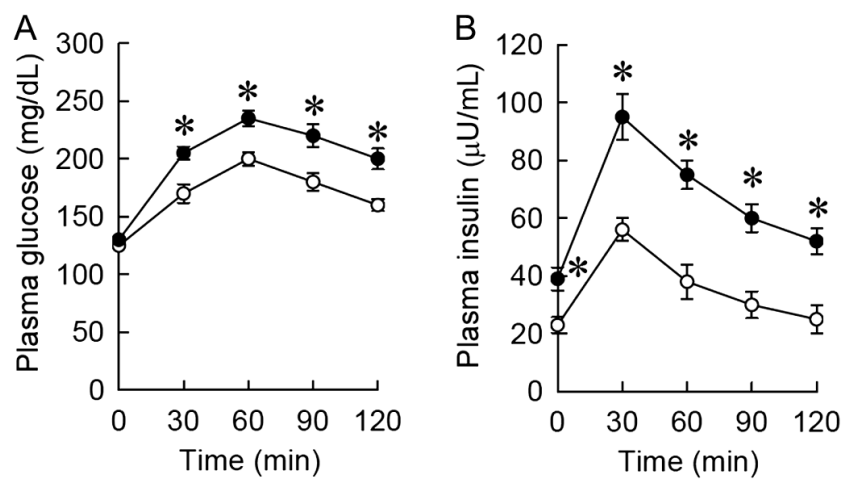

\section{Figure 1}

Changes in plasma glucose (A) and insulin levels (B) after oral glucose challenge in high-fat diet-fed rats (HF: $\bullet$ ) and the control (C: O). The results are the mean \pm S.D. for eight rats. ${ }^{*} P<0.05$ compared with the control.

Published by Bioscientifica Ltd. 

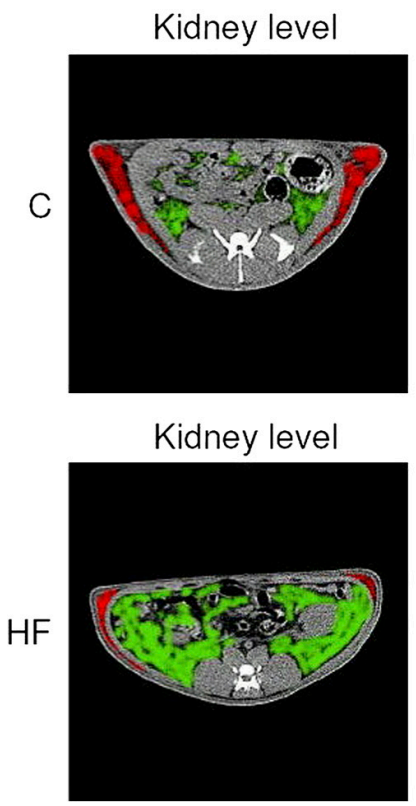

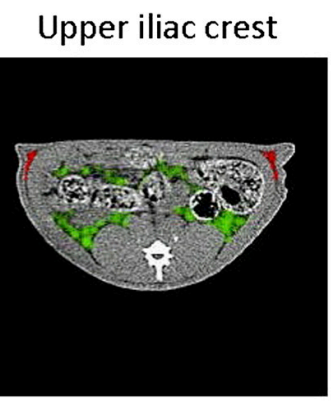

Upper iliac crest

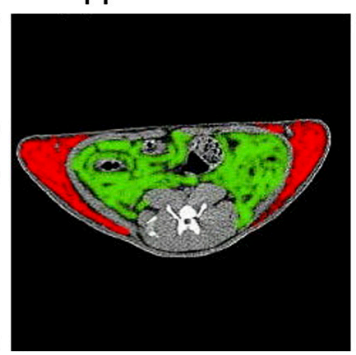

Inguinal level

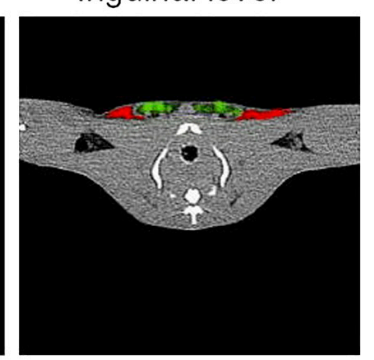

Inguinal level

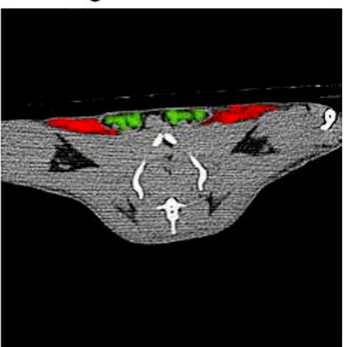

\section{Figure 2}

Body fat distribution in HF diet-fed rats (HF) and the control (C) determined by computed tomography (CT) at ultra-high resolution of the kidney, upper iliac crest and inguinal levels. The changes in the subcutaneous fat area (red color) and visceral fat area (green color) were measured by CT scan. A full color version of this figure is available at $h t t p: / / d x . d o i . o r g / 10.1530 /$ JOE-16-0064.
The reactions were performed at $95^{\circ} \mathrm{C}$ for $10 \mathrm{~min}$, followed by 40 cycles at $95^{\circ} \mathrm{C}$ for $15 \mathrm{~s}$ and $60^{\circ} \mathrm{C}$ for $1 \mathrm{~min}$.

\section{Statistical analysis}

The cell studies were performed four times. In the animal experiments, each group consisted of eight rats. The results were expressed as the mean \pm standard deviation (s.D.). Statistical significance was assessed by one-way analysis of variance or the Mann-Whitney $U$ test. A $P$-value of $<0.05$ was considered statistically significant.

\section{Results}

\section{Insulin resistance in HF diet-fed rats}

As shown in Table 1, the body weight increased significantly after 3 months in rats fed the HF diet compared with those fed the standard chow (C). In addition, the fasting levels of plasma glucose, insulin, triglycerides and cholesterol were significantly higher in the HF diet group than those in the control. To further evaluate the change in insulin sensitivity, an OGTT was performed, and the results for the two groups at the end of the last experimental week are shown in Fig. 1. There was no difference in the baseline plasma glucose level between the two groups; however, the plasma glucose level increased significantly in rats of the HF diet group compared with that in rats of the control at 30,60, 90 and $120 \mathrm{~min}$ after oral glucose challenge (Fig. 1A;
$P<0.05)$. As shown in Fig. $1 \mathrm{~B}$, the HF diet-fed rats had a significantly higher baseline plasma insulin level than the standard chow-fed rats. In response to oral glucose challenge, both groups responded with an increase in the plasma insulin level, except that the increase in the HF diet-fed rats was significantly higher than that in the standard chow-fed rats $(P<0.05)$.

\section{Visceral fat predominance in HF diet-fed rats}

As shown in Table 1, the weights of the epididymal, retroperitoneal and inguinal adipose deposits were significantly higher in rats of the HF diet group than those in rats of the control. The fat content of each area in the two groups was measured as the percentage of the total area occupied by fat as assessed by CT. As shown in Fig. 2 and Table 2, the fat content in the HF diet group was significantly higher than that in the control. Figure 3A shows hematoxylin and

Table 2 Visceral fat content measured by CT at the kidney, upper iliac crest and inguinal levels in controls (C) and high-fat diet-fed rats (HF) $(n=8)$.

\begin{tabular}{|c|c|c|c|}
\hline Locations & C & HF & $P$-value \\
\hline Kidney level & $31.4 \pm 1.9$ & $52.3 \pm 4.3$ & $<0.05$ \\
\hline Upper iliac crest level & $19.7 \pm 1.5$ & $29.3 \pm 1.3$ & $<0.05$ \\
\hline Inguinal level & $5.6 \pm 0.4$ & $7.6 \pm 0.4$ & $<0.05$ \\
\hline
\end{tabular}

The units are the percentage of the total cross-sectional area. Values are expressed as mean \pm S.D. Statistical analysis was performed using Mann-Whitney $U$ test with repeated measurements. A value of $P<0.05$ was considered significant.

Published by Bioscientifica Ltd 
A

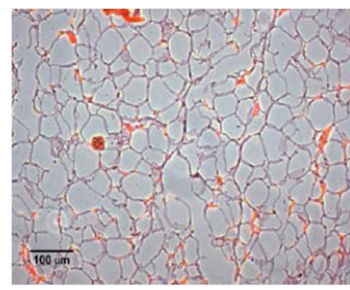

C

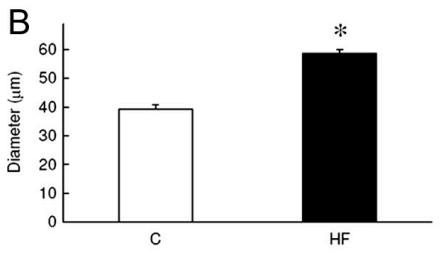

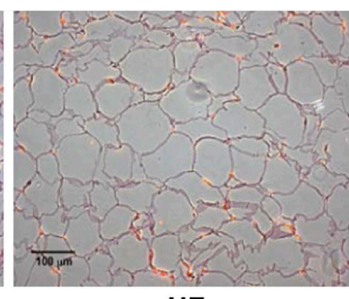

HF

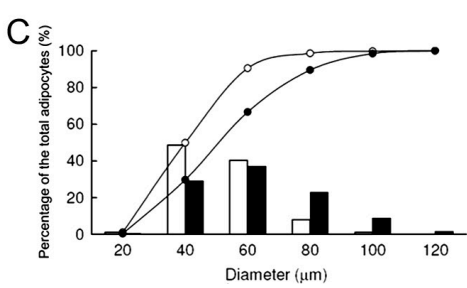

D
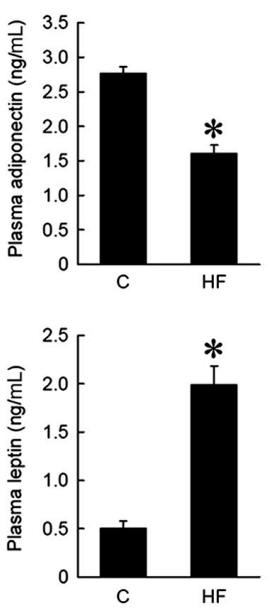
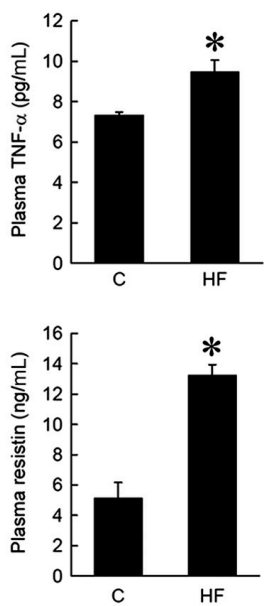

\section{Figure 3}

Histology of adipose tissue from HF diet-fed rats (HF) and the control (C). (A) Hematoxylin and eosin staining of epididymal white adipose tissue from the control and the HF diet group. (B) Epididymal adipocyte diameter in the HF diet-fed rats and the control (C); in each group, the diameters were measured in each of eight rats, and the results are the mean \pm S.D. ${ }^{*} P<0.05$ compared with the control. (C) Epididymal adipocyte size distribution in $\mathrm{HF}$ diet-fed rats (HF: $\square, \square$ ) and the control (C: $O, \square$ ); in each group, adipocytes from eight rats were pooled. (D) Plasma adiponectin, leptin, resistin and TNF- $\alpha$ levels in the HF diet-fed rats and the control (C); in each group, plasma data were measured in each of eight rats, and the results are the mean \pm S.D. ${ }^{*} P<0.05$ compared with the control. A full color version of this figure is available at http://dx.doi.org/10.1530/JOE-16-0064.

eosin-stained slides of the epididymal white adipose tissue. The mean adipocyte size was significantly greater in the HF diet-fed rats than that in the control (Fig. 3B), and the adipocyte size distribution curve shifted to the right in the HF diet-fed rats compared with that in the control (Fig. 3C). We also examined the levels of plasma adipocytokines in both groups and showed a decreased plasma adiponectin level and increased levels of plasma leptin, resistin and TNF- $\alpha$ in HF diet-fed rats compared with those in the control (Fig. 3D; $P<0.05$ ). From these data, we further investigated the interplay between these factors and glomerular podocytes.

\section{Renal function impairment and podocyte injury in HF diet-fed rats}

Table 1 shows that the kidney weight increased significantly in HF diet-fed rats compared with that in the control, as well as the levels of plasma creatinine (but not BUN), 24 h urinary protein excretion, creatinine and the protein/creatinine ratio. The immunohistochemical staining for desmin in the glomerulus and tubules showed much more brown pigmentation in the HF diet group than the control (Fig. 4A), and the optical density of the desmin staining in the glomerulus was significantly higher in the HF diet group (Fig. 4B, right panel). The histopathological staining for desmin showed an increase in glomerular damage in the HF diet group, but not in the control (Fig. 4A), and the calculated glomerular volume was also significantly higher in the HF diet group than that in the control (Fig. 4B, left panel). We also measured the levels of desmin, CD2AP and nephrin in the kidney by Western blotting. As shown in Fig. 4C, increased desmin expression, but decreased CD2AP and nephrin expression, was seen in the HF diet group compared with that in the control. When the renal cortical tissue was examined by TEM, obvious podocyte effacement was observed in the HF diet group, but not in the control (Fig. 4D). Because desmin is an injury marker, increased desmin expression associated with glomerular damage (Herrmann et al. 2012) and the downregulation of CD2AP and nephrin indicated podocyte injury in the kidney (Asanuma et al. 2007, Xiao et al. 2013).

\section{Increased desmin expression and decreased CD2AP and nephrin expression in ACM-treated podocytes}

Based on the Western blotting results, we found that the high-fat diet damaged podocytes. Therefore, we cultured mature podocytes and examined the effects of PCM and ACM with phase photography and immunofluorescent staining (Fig. 5A). We found ACM disrupted the cytoskeleton in podocytes. We also incubated podocytes for $24 \mathrm{~h}$ with PCM or ACM with/without the IgG isotype and an antibody against adiponectin, leptin, resistin or 
A
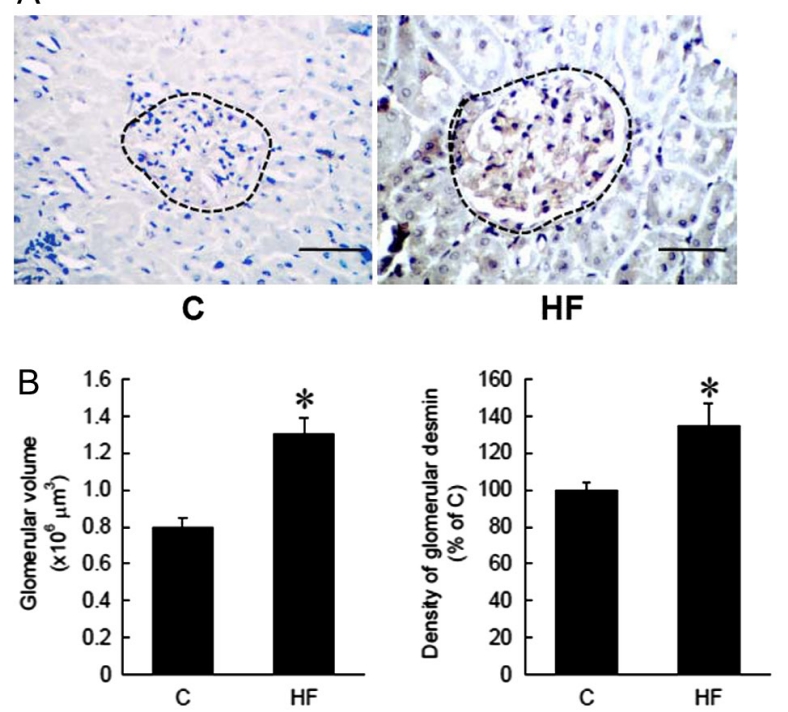

C
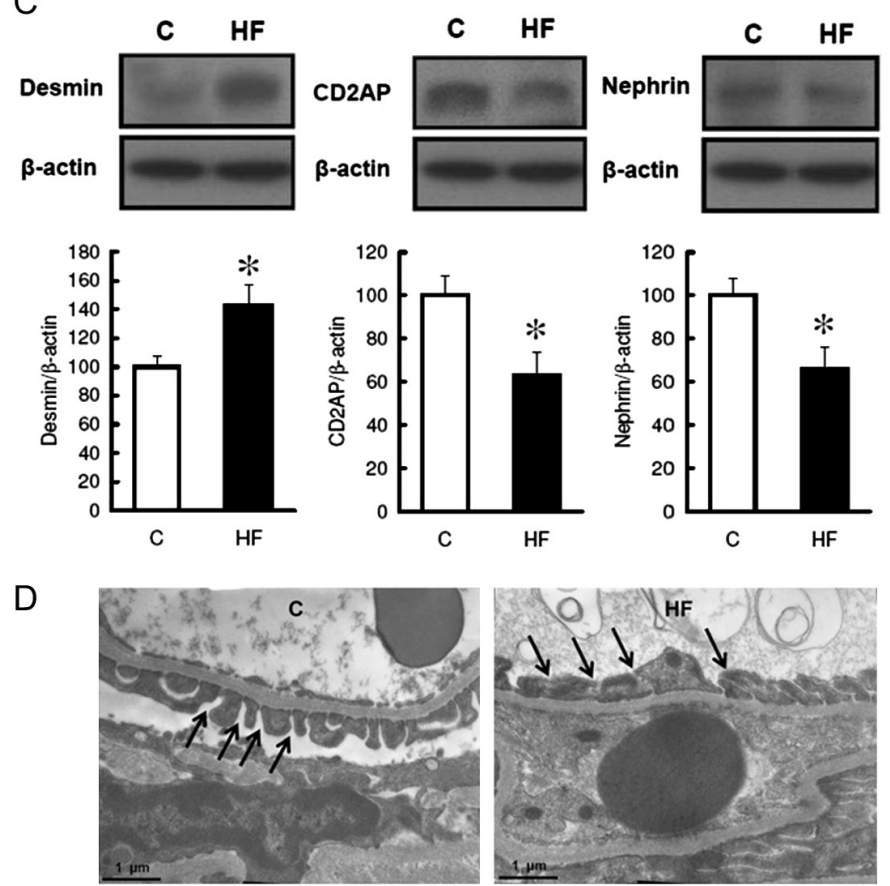

Figure 4

(A) Representative images of sections of the glomerulus immunohistochemically stained for desmin (brown stain) from the control (C) and HF diet-fed rats (HF). Glomerular hypertrophy and increased desmin staining in the glomerulus (white arrowhead) and tubules (white arrow) in the HF diet group; scale bar, $50 \mu \mathrm{m}$. (B) Calculated glomerular volume (left panel) and the optical density of glomerular desmin (right panel) in the control and HF diet group. Glomerular volume was calculated as the total area of the glomerular tuft $\left(\mathrm{A}_{T}\right) \times(1.5) \times 1.382 / 1.01(1.382=$ shape coefficient, $1.01=$ size distribution coefficient). (C) Expression of desmin, CD2AP and nephrin in the kidney cortex from the control (C) and high-fat diet-fed rats (HF) normalized to the $\beta$-actin level. In $B$ and $C$, the results are the mean \pm s.D. for eight rats. $* P<0.05$ compared with the control. (D) Podocyte effacement (indicated by arrows) in the HF diet group compared with the control (magnification, 15,000x). A full color version of this figure is available at http://dx.doi.org/10.1530/ JOE-16-0064.

TNF- $\alpha$. We examined the effects of adipocyte-derived factors on podocyte function and measured the mRNA levels of desmin, CD2AP and nephrin by quantitative RT-PCR. As shown in Fig. 5B, increased desmin mRNA expression and decreased CD2AP and nephrin mRNA expression were seen in the ACM-treated group compared with that in the PCM-treated group $(P<0.05)$. We also found that antibodies against leptin, resistin and TNF- $\alpha$ in the ACM-treated group were the three major correction factors, and the antibody against adiponectin in the ACM-treated group was a worse factor $(P<0.05)$ compared with those in the ACM-treated group alone. Moreover, we also observed desmin, CD2AP and nephrin immunostaining of PCM-cultured and ACM-cultured podocytes with/without the IgG isotype as the control or with an antibody against adiponectin, leptin or resistin, or TNF- $\alpha$. As shown in Fig. 5C, D and E, antibodies against resistin, TNF- $\alpha$ and leptin significantly decreased desmin expression and increased CD2AP and nephrin expression in ACM-treated podocytes compared with that in the ACM-treated group alone $(P<0.05)$.

\section{Discussion}

Excess visceral fat is an independent cardiovascular risk factor (Matsushita et al. 2014, Okamoto et al. 2014). In this study, the HF diet induced obesity, insulin resistance, hyperinsulinemia and dyslipidemia in rats. A significant increase in visceral fat was seen in the HF diet group (Fig. 2 and Table 2). In addition, male Sprague Dawley rats fed a HF diet for 12 weeks in our study developed morphological and functional kidney disorders as same as past articles (Pinhal et al. 2013, Jenkin et al. 2016). Previous studies showed that obesity carries an increased risk of CKD and ESRD (Gelber et al. 2005, Obermayr et al. 2008, Satirapoj et al. 2013), and a recent report showed that perirenal visceral adiposity strongly associates with renal inflammation and diabetic nephropathy (Ndisang et al. 2014). Our data demonstrated that the levels of plasma creatinine, $24 \mathrm{~h}$ urinary protein and creatinine excretion and the protein/creatinine ratio were significantly elevated in the HF diet group: glomerular changes that are consistent with kidney injury. Moreover, the visceral

Published by Bioscientifica Ltd 

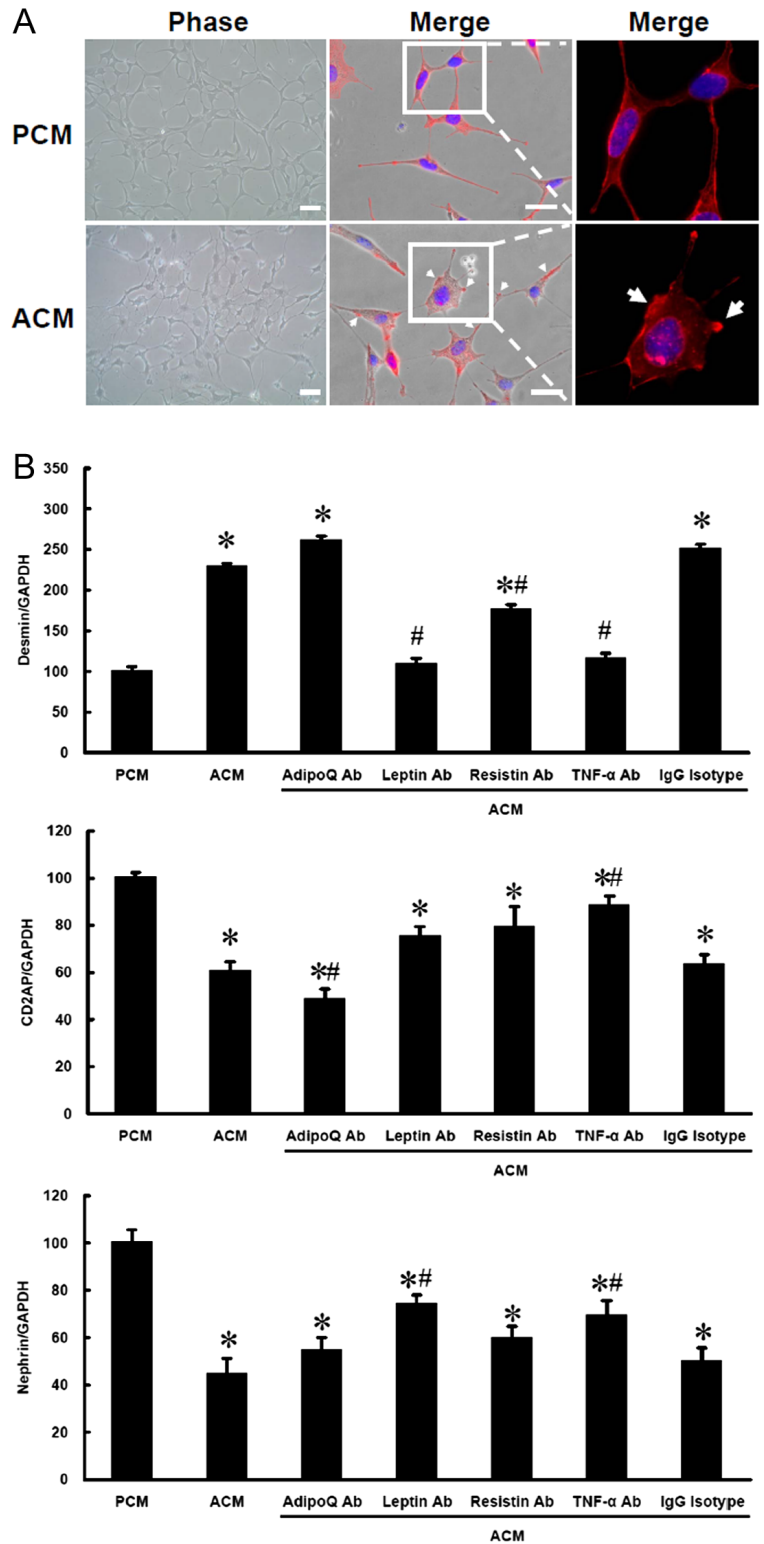
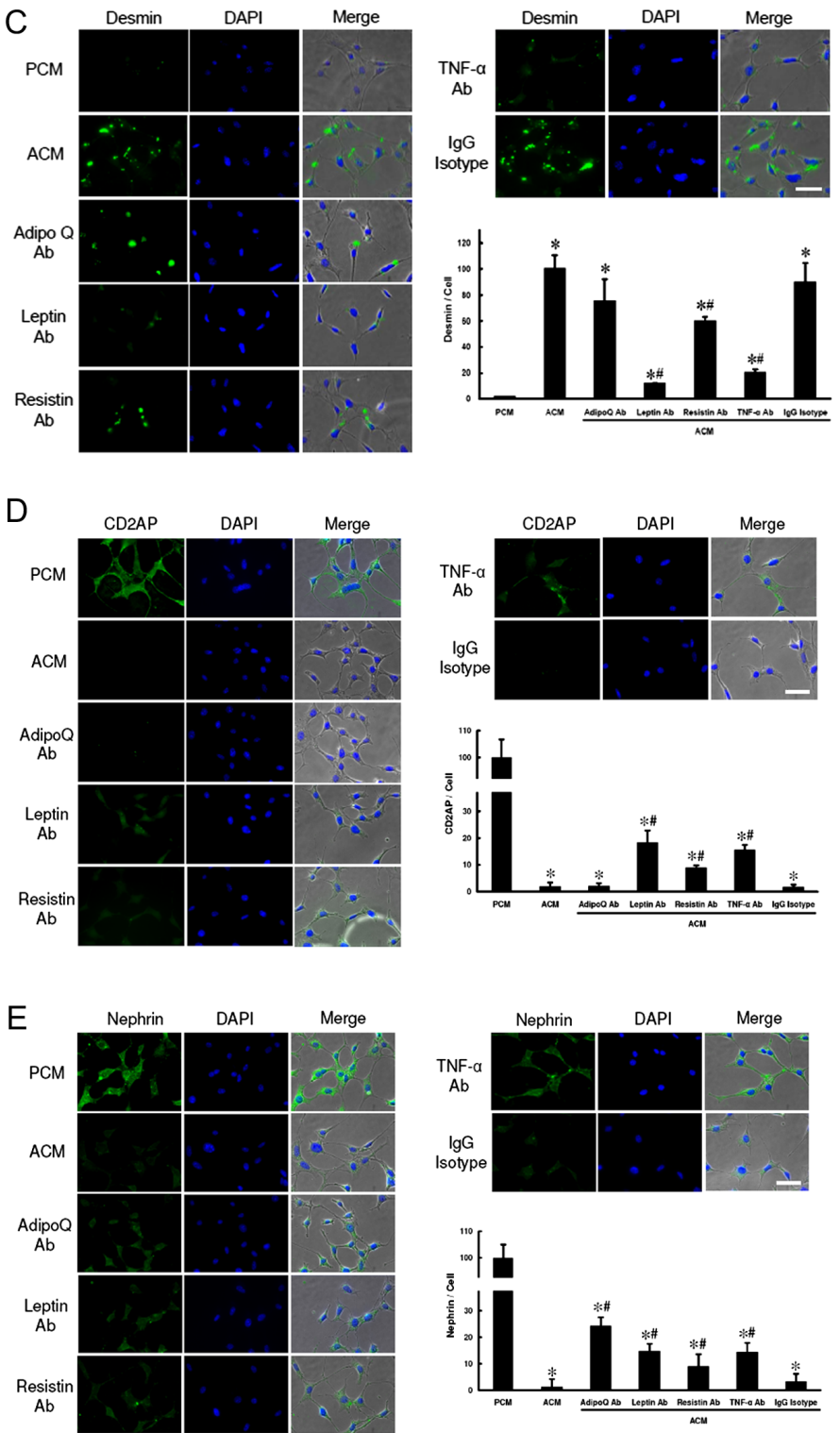

Figure 5

Effects of preadipocyte-conditioned medium (PCM) or adipocyte-conditioned medium (ACM) on cultured differentiated podocytes. (A) Phase photography and immunofluorescent staining of F-actin (red) in podocytes cultured with PCM and ACM demonstrated a disruption of the podocyte cytoskeleton (white arrow) after treatment with ACM. Cell nuclei were stained with DAPI (blue); scale bar, $50 \mu \mathrm{m}$. (B) Well-differentiated podocytes were incubated for $24 \mathrm{~h}$ with PCM or ACM, as well as with/without the IgG isotype as the control and an antibody against adiponectin, leptin, resistin or TNF- $\alpha$. The expression of desmin, CD2AP and nephrin was measured by RT-PCR and normalized to the GAPDH level. (C, D and E) Cultured podocytes on $12 \mathrm{~mm}$ cover slips in a 24-well plate were incubated for $24 \mathrm{~h}$ with PCM or ACM, as well as with/without the IgG isotype as the control or with an antibody against adiponectin, leptin, resistin or TNF- $\alpha$. The immunofluorescent staining of desmin (green), CD2AP (green) and nephrin (green) were evaluated; scale bar, $50 \mu \mathrm{m}$. The results are the mean \pm s.D. for four independent experiments. ${ }^{*} P<0.05$ compared with $P C M$ treatment. $\# P<0.05$ compared with ACM treatment. A full color version of this figure is available at http://dx.doi.org/10.1530/JOE-16-0064.

fat level also associates with coronary artery calcification in nondialysis-dependent CKD patients (Cordeiro et al. 2013). Our results suggested that increased visceral adiposity might enhance renal damage and cardiovascular risk in obese individuals. The limitation of this study was the small number of rats, which was not indicative of a normal distribution, and we plan on using more animals in future studies.

Past research has manifested morphological and histological changes of obesity related to glomerulopathy, such as glomerular hypertrophy with/without focal segmental glomerulosclerosis (Kambham et al. 2001, 
Chen et al. 2008). In addition, there is other evidence of obesity-related glomerulopathy demonstrating vacuolar degeneration in renal tubules, increased glomerular ECM accumulation (Pan et al. 2014) and increased expression of TGF $\beta 1$, AT1 receptor, transcription factor ZEB2, fibronectin and desmin in glomerular and tubular cells (Pinhal et al. 2013). In this study, we focused on changes in the glomerulus because of its important physiological function as a barrier in humans. Our data indicated increased proteinuria, glomerular hypertrophy, increased desmin expression in the glomerulus and tubules and decreased CD2AP and nephrin expression of the glomerulus in $\mathrm{HF}$ diet-fed rats. Resveratrol and $\mathrm{CB}_{2}$ receptor agonism were also studied to alleviate renal injury in diet-induced obesity rats (Jenkin et al. 2016, Pan et al. 2014). Unlike previous studies, we hypothesized that adipocyte-derived factors act on podocytes in vitro and tried to search for the individual effect of each adipocytederived factor on podocytes.

Ndisang and coworkers (2014) showed increased proinflammatory/oxidativemediatorlevels, macrophage infiltration and profibrotic/extracellular matrix protein levels in perirenal adipose tissue, and decreased renal function in Zucker diabetic fatty rats after using hemin (a heme oxygenase inducer) to reduce perirenal visceral adiposity, renal inflammation and diabetic nephropathy. Knight and coworkers (2010) showed that the expression of glomerular nephrin expression decreases and the urinary albumin level increases in a HF diet model of obesity and hypertension. In this study, we observed renal cortical tissue injury, which was observed as an increase in desmin expression and a decrease in CD2AP and nephrin expression, in the HF diet-fed Sprague Dawley rats. Results of TEM revealed obvious podocyte effacement in the HF diet group. Our results showed that the development of impaired renal function associated with increased visceral fat levels, demonstrating the presence of podocyte slit diaphragm damage, as shown by the decreased expression of CD2AP and nephrin (Fig. 5), indicators of glomerular damage and the main manifestation of kidney injury. Further studies are needed to validate the relationship between perirenal visceral adiposity and podocyte slit diaphragm injury. In this study, we only studied the outer portion of the kidney cortex, and further studies are needed to investigate the zonal variations in the juxtaglomerular area.

High adipocytokine levels associate with insulin resistance, low-grade inflammation, endothelial dysfunction and vascular damage, and they show a positive correlation with CKD (De Cosmo et al. 2013). Adiponectin has a protective effect against the development of albuminuria in a recent animal study (Christou \& Kiortsis 2014). Moreover, the serum adiponectin concentration is independently associated with albuminuria in CKD patients (Kim et al. 2016, Ortega Moreno et al. 2015). Increased leptin concentrations correlate with renal injury and proteinuria, and also with damaged cultured renal proximal tubule cells in in vitro experiments (Briffa et al. 2015, Thieme \& OliveiraSouza 2015). The serum resistin level plays an important role in modulating kidney function in nondiabetic, untreated individuals (Menzaghi et al. 2012). Leptin and resistin associate with the early stages of CKD in obese patients (Stępień et al. 2013). Elevated serum resistin levels exhibit higher urinary albumin excretion in nondiabetic patients with essential hypertension (Tsioufis et al. 2010). From these studies, the interplay between adipocytokines and proteinuria remains unclear. In the cultured podocyte experiments, we demonstrated that the treatment of mature podocytes with adipocyteconditioned medium resulted in increased desmin expression and decreased CD2AP and nephrin expression compared with podocytes treated with PCM, especially enriched with leptin, resistin and TNF- $\alpha$ (Fig. 5B). Furthermore, decreased protein expression of desmin and increased protein expression of CD2AP and nephrin were observed by immunostaining after resistin, leptin and TNF- $\alpha$ antibody treatment of the ACM group (Fig. 5C, D and E), consistent with RT-PCR results. These findings showed that adipocyte-derived factor(s) can affect structural protein expression in podocytes, which impairs the function of the renal defense barrier. We also provided evidence that the adipocyte factors may play a pathological role in obesity-associated podocytopathy. Our data show that adipocyte-derived factors were very harmful to podocytes. Adipocytes, therefore, play a pivotal role in the pathogenesis of podocytopathy. In conclusion, our results revealed that adipocytes may play an etiological role in the development of obesity-related podocytopathy, and adipocytokines may be critical in the pathogenesis of obesity-related podocytopathy.

\section{Declaration of interest}

The authors declare that there is no conflict of interest that could be perceived as prejudicing the impartiality of the research reported.

\section{Funding}

This study was supported by research grants from the Ministry of Science and Technology (MOST 105-3011-B-010-001; 1-3-2), the Department of

Published by Bioscientifica Ltd. 
Health of the Taipei City Government (No. 10001-62-043) and from the Ministry of Education, Aim for the Top University Plan, Taipei, Taiwan.

\section{Author contribution statement}

The study was conceived and designed by J $Y C$ and $C C J$. The experiments and data collection were performed by J Y C, Y W C, C C L, Y T L, C H T, L K C, T C H, H M H, Y T W, T T K, L Y W and S W H. Data analysis and interpretation were performed by J Y C, D Y J, L K C, L Y W and C C J. All authors were involved in writing the paper and approved the submitted and published versions.

\section{Acknowledgements}

We thank Professor Jochen Reiser, MD, PhD (Rush University Medical Center, Chicago, IL, USA), for kindly providing the conditionally immortalized mouse podocytes.

\section{References}

Adamczak M, Gross ML, Krtil J, Koch A, Tyralla K, Amann K \& Ritz E 2003 Reversal of glomerulosclerosis after high-dose enalapril treatment in subtotally nephrectomized rats. Journal of the American Society of Nephrology 14 2833-2842. (doi:10.1097/01. ASN.0000095248.91994.D3)

Asanuma K, Campbell KN, Kim K, Faul C \& Mundel P 2007 Nuclear relocation of the nephrin and CD2AP-binding protein dendrin promotes apoptosis of podocytes. PNAS $\mathbf{1 0 4} 10134-10139$. (doi:10.1073/pnas.0700917104)

Briffa JF, Grinfeld E, Mathai ML, Poronnik P, McAinch AJ \& Hryciw DH 2015 Acute leptin exposure reduces megalin expression and upregulates TGF $\beta 1$ in cultured renal proximal tubule cells. Molecular and Cellular Endocrinology 401 25-34. (doi:10.1016/j. mce.2014.11.024)

Canpolat N, Caliskan S, Sever L, Guzeltas A, Kantarci F, Candan C, Civilibal M, Kasapcopur O \& Arisoy N 2012 Glucose intolerance: is it a risk factor for cardiovascular disease in children with chronic kidney disease? Pediatric Nephrology 27 627-635. (doi:10.1007/s00467011-2034-3)

Cerezo C, Segura J, Praga M \& Ruilope LM 2013 Guidelines updates in the treatment of obesity or metabolic syndrome and hypertension. Current Hypertension Reports 15 196-203. (doi:10.1007/s11906-0130337-4)

Chen HM, Li SJ, Chen HP, Wang QW, Li LS \& Liu ZH 2008 Obesity-related glomerulopathy in China: a case series of 90 patients. American Journal of Kidney Diseases 52 58-65. (doi:10.1053/j. ajkd.2008.02.303)

Christou GA \& Kiortsis DN 2014 The role of adiponectin in renal physiology and development of albuminuria. Journal of Endocrinology 221 R49-R61. (doi:10.1530/JOE-13-0578)

Cordeiro AC, Qureshi AR, Lindholm B, Amparo FC, Tito-Paladino-Filho A, Perini M, Lourenço FS, Pinto IM, Amodeo C \& Carrero JJ 2013 Visceral fat and coronary artery calcification in patients with chronic kidney disease. Nephrology Dialysis Transplantation 28 (Supplement 4) iv152-iv159. (doi:10.1093/ndt/gft250)

De Cosmo S, Menzaghi C, Prudente S \& Trischitta V 2013 Role of insulin resistance in kidney dysfunction: insights into the mechanism and epidemiological evidence. Nephrology Dialysis Transplantation 28 29-36. (doi:10.1093/ndt/gfs290)

Després JP, Lemieux I, Bergeron J, Pibarot P, Mathieu P, Larose E, Rodés-Cabau J, Bertrand OF \& Poirier P 2008 Abdominal obesity and the metabolic syndrome: contribution to global cardiometabolic risk. Arteriosclerosis, Thrombosis, and Vascular Biology 28 1039-1049. (doi:10.1161/ATVBAHA.107.159228)
Foster MC, Hwang SJ, Larson MG, Lichtman JH, Parikh NI, Vasan RS, Levy D \& Fox CS 2008 Overweight, obesity, and the development of stage 3 CKD: the Framingham Heart Study. American Journal of Kidney Diseases 52 39-48. (doi:10.1053/j.ajkd.2008.03.003)

Fukuda A, Wickman LT, Venkatareddy MP, Sato Y, Chowdhury MA, Wang SQ, Shedden KA, Dysko RC, Wiggins JE \& Wiggins RC 2012 Angiotensin II-dependent persistent podocyte loss from destabilized glomeruli causes progression of end stage kidney disease. Kidney International 81 40-55. (doi:10.1038/ki.2011.306)

Galic S, Oakhill JS \& Steinberg GR 2010 Adipose tissue as an endocrine organ. Molecular and Cellular Endocrinology 316 129-139. (doi:10.1016/j.mce.2009.08.018)

Gelber RP, Kurth T, Kausz AT, Manson JE, Buring JE, Levey AS \& Gaziano JM 2005 Association between body mass index and CKD in apparently healthy men. American Journal of Kidney Diseases $\mathbf{4 6}$ 871-880. (doi:10.1053/j.ajkd.2005.08.015)

Heo NJ, Ahn JM, Lee TW, Chin HJ, Na KY, Chae DW \& Kim S 2010 Very low-grade albuminuria reflects susceptibility to chronic kidney disease in combination with cardiovascular risk factors. Hypertension Research 33 573-578. (doi:10.1038/hr.2010.39)

Herrmann A, Tozzo E \& Funk J 2012 Semi-automated quantitative image analysis of podocyte desmin immunoreactivity as a sensitive marker for acute glomerular damage in the rat puromycin aminonucleoside nephrosis (PAN) model. Experimental and Toxicologic Pathology 64 45-49. (doi:10.1016/j.etp.2010.06.004)

Hillebrand JJ, Langhans W \& Geary N 2010 Validation of computed tomographic estimates of intra-abdominal and subcutaneous adipose tissue in rats and mice. Obesity 18 848-853. (doi:10.1038/ oby.2009.341)

Hsu CY, Iribarren C, McCulloch CE, Darbinian J \& Go AS 2009 Risk factors for end-stage renal disease: 25-year follow-up. Archives of Internal Medicine 169 342-350. (doi:10.1001/ archinternmed.2008.605)

Hung AM \& Ikizler TA 2011 Factors determining insulin resistance in chronic hemodialysis patients. Contributions to Nephrology $\mathbf{1 7 1}$ 127-134. (doi:10.1159/000327177)

Jenkin KA, O'Keefe L, Simcocks AC, Briffa JF, Mathai ML, McAinch AJ \& Hryciw DH 2016 Renal effects of chronic pharmacological manipulation of $\mathrm{CB}_{2}$ receptors in rats with diet-induced obesity. British Journal of Pharmacology 173 1128-1142. (doi:10.1111/bph.13056)

Kakimoto T, Okada K, Hirohashi Y, Relator R, Kawai M, Iguchi T, Fujitaka K, Nishio M, Kato T, Fukunari A, et al. 2014 Automated image analysis of a glomerular injury marker desmin in spontaneously diabetic Torii rats treated with losartan. Journal of Endocrinology 222 43-51. (doi:10.1530/JOE-14-0164)

Kambham N, Markowitz GS, Valeri AM, Lin J \& D'Agati VD 2001 Obesity-related glomerulopathy: an emerging epidemic. Kidney International 59 1498-1509. (doi:10.1046/j.15231755.2001.0590041498.x)

Kim HY, Bae EH, Ma SK, Chae DW, Choi KH, Kim YS, Hwang YH, Ahn C \& Kim SW 2016 Association of serum adiponectin level with albuminuria in chronic kidney disease patients. Clinical and Experimental Nephrology 20 443-449. (doi:10.1055/s-2008-1068326)

Knight SF, Yuan J, Roy S \& Imig JD 2010 Simvastatin and tempol protect against endothelial dysfunction and renal injury in a model of obesity and hypertension. American Journal of Physiology: Renal Physiology 298 F86-F94. (doi:10.1152/ajprenal.00351.2009)

Kümpers P, Gueler F, Rong S, Mengel M, Tossidou I, Peters I, Haller H \& Schiffer M 2007 Leptin is a coactivator of TGF-beta in unilateral ureteral obstructive kidney disease. American Journal of Physiology: Renal Physiology 293 F1355-F1362. (doi:10.1152/ ajprenal.00003.2007)

Levey AS, Bosch JP, Lewis JB, Greene T, Rogers N \& Roth D 1999 A more accurate method to estimate glomerular filtration rate from serum creatinine: a new prediction equation. Modification of Diet in Renal Disease Study Group. Annals of Internal Medicine 130 461-470. 
Locatelli F, Pozzoni P \& Del Vecchio L 2006 Renal manifestations in the metabolic syndrome. Journal of the American Society of Nephrology $\mathbf{1 7}$ (Supplement 2) S81-S85. (doi:10.1681/asn.2005121332)

Mathieson PW 2011 The podocyte as a target for therapies - new and old. Nature Reviews Nephrology 8 52-56. (doi:10.1038/nrneph.2011.171)

Matsushita Y, Nakagawa T, Yamamoto S, Kato T, Ouchi T, Kikuchi N, Takahashi Y, Yokoyama T, Mizoue T \& Noda M 2014 Adiponectin and visceral fat associate with cardiovascular risk factors. Obesity 22 287-291. (doi:10.1002/oby.20425)

Menzaghi C, Salvemini L, Fini G, Thompson R, Mangiacotti D, Di Paola R, Morini E, Giorelli M, De Bonis C, De Cosmo S, et al. 2012 Serum resistin and kidney function: a family-based study in non-diabetic, untreated individuals. PLOS ONE 7 e38414. (doi:1.1371/ journal.pone.0038414)

Mills KT, Hamm LL, Alper AB, Miller C, Hudaihed A, Balamuthusamy S, Chen CS, Liu Y, Tarsia J, Rifai N, et al. 2013 Circulating adipocytokines and chronic kidney disease. PLOS ONE 8 e76902. (doi:10.1371/journal.pone.0076902)

Mundel P, Reiser J, Zúñiga Mejía Borja A, Pavenstädt H, Davidson GR, Kriz W \& Zeller R 1997 Rearrangements of the cytoskeleton and cell contacts induce process formation during differentiation of conditionally immortalized mouse podocyte cell lines. Experimental Cell Research 236 248-258. (doi:10.1006/excr.1997.3739)

Munkhaugen J, Lydersen S, Widerøe TE \& Hallan S 2009 Prehypertension, obesity, and risk of kidney disease: 20-year follow-up of the HUNT I study in Norway. American Journal of Kidney Diseases 54 638-646. (doi:10.1053/j.ajkd.2009.03.023)

Nanayakkara PW, Le Poole CY, Fouque D, van Guldener C, Stehouwer CD, Smulders YM, van Ittersum FJ, Siegert CE, Drai J, Kostense PJ, et al. 2009 Plasma adiponectin concentration has an inverse and a non linear association with estimated glomerular filtration rate in patients with K/DOQI 3-5 chronic kidney disease. Clinical Nephrology 72 21-30. (doi:10.5414/cnp72021)

Ndisang JF, Jadhav A \& Mishra M 2014 The heme oxygenase system suppresses perirenal visceral adiposity, abates renal inflammation and ameliorates diabetic nephropathy in Zucker diabetic fatty rats. PLOS ONE 9 e87936. (doi:10.1371/journal.pone.0087936. eCollection2014)

Nguyen S \& Hsu CY 2007 Excess weight as a risk factor for kidney failure. Current Opinion in Nephrology and Hypertension 16 71-76. (doi:10.1210/jc.2005-2143)

Ninomiya T \& Kiyohara Y 2007 Albuminuria and chronic kidney disease in association with the metabolic syndrome. Journal of the CardioMetabolic Syndrome 2 104-107. (doi:10.1111/j.1559-4564.2007.05734.x)

Obermayr RP, Temml C, Knechtelsdorfer M, Gutjahr G, Kletzmayr J, Heiss S, Ponholzer A, Madersbacher S, Oberbauer R \& Klauser-Braun R 2008 Predictors of new-onset decline in kidney function in a general middle-European population. Nephrology Dialysis Transplantation 23 1265-1273. (doi:10.1093/ndt/gfm790)

Okamoto T, Morimoto S, Ikenoue T, Furumatsu Y \& Ichihara A 2014 Visceral fat level is an independent risk factor for cardiovascular mortality in hemodialysis patients. American Journal of Nephrology 39 122-129. (doi:10.1159/000358335)

Ortega Moreno L, Lamacchia O, Copetti M, Salvemini L, De Bonis C, De Cosmo S, Cignarelli M, Trischitta V \& Menzaghi C 2015 Serum adiponectin and glomerular filtration rate in patients with type 2 diabetes. PLOS ONE 10 e0140631. (doi:10.1371/journal.pone.0140631)

Pan QR, Ren YL, Zhu JJ, Hu YJ, Zheng JS, Fan H, Xu Y, Wang G \& Liu WX 2014 Resveratrol increases nephrin and podocin expression and alleviates renal damage in rats fed a high-fat diet. Nutrients 6 2619-2631. (doi:10.3390/nu6072619)

Pham H, Utzschneider KM \& de Boer IH 2011 Measurement of insulin resistance in chronic kidney disease. Current Opinion in Nephrology and Hypertension 20 640-646. (doi:10.1097/MNH.0b013e32834b23c1)

Phillips LK \& Prins JB 2008 The link between abdominal obesity and the metabolic syndrome. Current Hypertension Reports 10 156-164. (doi:10.1007/s11906-008-0029-7)

Pinhal CS, Lopes A, Torres DB, Felisbino SL, Rocha Gontijo JA \& Boer PA 2013 Time-course morphological and functional disorders of the kidney induced by long-term high-fat diet intake in female rats. Nephrology Dialysis Transplantation 28 2464-2476. (doi:10.1093/ndt/ gft304)

Satirapoj B, Supasyndh O, Mayteedol N, Punpanich D, Chaiprasert A, Nata N, Ruangkanchanasetr P, Kanjanakul I \& Choovichian P 2013 Obesity and its relation to chronic kidney disease: a populationbased, cross-sectional study of a Thai army population and relatives. Nephrology 18 229-234. (doi:10.1111/nep.12023)

Seow KM, Hwang JL, Wang PH, Ho LT \& Juan CC 2011 Expression of visfatin mRNA in peripheral blood mononuclear cells is not correlated with visfatin mRNA in omental adipose tissue in women with polycystic ovary syndrome. Human Reproduction 26 2869-2873. (doi:10.1093/humrep/der267)

Sharma K 2009 The link between obesity and albuminuria: adiponectin and podocyte dysfunction. Kidney International 76 145-148. (doi:10.1038/ki.2009.137)

Sharma K, Ramachandrarao S, Qiu G, Usui HK, Zhu Y, Dunn SR, Ouedraogo R, Hough K, McCue P, Chan L, et al. 2008 Adiponectin regulates albuminuria and podocyte function in mice. Journal of Clinical Investigation 118 1645-1656. (doi:10.1172/JCI32691)

Stępień M, Stępień A, Wlazeł RN, Paradowski M, Banach M, Rysz M \& Rysz J 2013 Obesity indices and adipokines in non-diabetic obese patients with early stages of chronic kidney disease. Medical Science Monitor 19 1063-1072. (doi:10.12659/MSM.889390)

Tharaux PL \& Huber TB 2012 How many ways can a podocyte die? Seminars in Nephrology 32 394-404. (doi:10.1016/j. semnephrol.2012.06.011)

Thieme K \& Oliveira-Souza M 2015 Renal hemodynamic and morphological changes after 7 and 28 days of leptin treatment: the participation of angiotensin II via the AT1 receptor. PLOS ONE $\mathbf{1 0}$ e0122265. (doi:10.1371/journal.pone.0122265)

Tsioufis C, Dimitriadis K, Selima M, Miliou A, Toutouzas K, Roussos D, Stefanadi E, Tousoulis D, Kallikazaros I \& Stefanadis C 2010 Association of resistin with urinary albumin excretion in nondiabetic patients with essential hypertension. American Journal of Hypertension 23 681-686. (doi:10.1038/ajh.2010.34.)

Van de Voorde J, Pauwels B, Boydens C \& Decaluwé K 2013 Adipocytokines in relation to cardiovascular disease. Metabolism 62 1513-1521. (doi:10.1016/j.metabol.2013.06.004)

Weil EJ, Lemley KV, Mason CC, Yee B, Jones LI, Blouch K, Lovato T, Richardson M, Myers BD \& Nelson RG 2012 Podocyte detachment and reduced glomerular capillary endothelial fenestration promote kidney disease in type 2 diabetic nephropathy. Kidney International $\mathbf{8 2}$ 1010-1017. (doi:10.1038/ki.2012.234)

Xiao Z, Rodriguez PQ, He L, Betsholtz C, Tryggvason K \& Patrakka J 2013 Wtip- and gadd45a-interacting protein dendrin is not crucial for the development or maintenance of the glomerular filtration barrier. PLOS ONE 8 e83133. (doi:10.1371/journal. pone.0083133)

Received in final form 29 July 2016

Accepted 18 August 2016

Accepted Preprint published online 18 August 2016 http://joe.endocrinology-journals.org

DOI: $10.1530 / \mathrm{JOE}-16-0064$
๑ 2016 Society for Endocrinology Printed in Great Britain 